\title{
Vie professionnelle et médias sociaux : le cas de l'URPP
}

L'implantation du web depuis les années 1990 a bouleversé la gestion de l'information, les échanges entre les personnes et les entreprises. Si le courriel demeure un véhicule de communication privilégié, les internautes recourent de plus en plus aux réseaux sociaux, aux blogues, aux wikis et autres outils web facilitant le travail en équipe ${ }^{1-8}$. Ces outils sont souvent utilisés avec succès par des organismes lors de campagnes médiatiques. Ils sont utilisés pour faciliter le réseautage professionnel et contribuent à la promotion d'initiatives de recherche avec des moyens relativement limités.

En ce qui concerne la vie professionnelle, les pharmaciens et médecins peuvent utiliser différents médias sociaux afin de 
réseauter. Par exemple, LinkedIn (LinkedIn Corporation, Mountain View [Californie], lancé en 2003), Facebook (Facebook Inc, Menlow Park [Californie], ouvert à tous depuis 2006), BiomedExperts (Elsevier Inc, Oxford [United Kingdom]) et plus récemment Google+ (Google, lancé en 2011) comprennent l'affichage d'un profil individuel ou professionnel, incluant la formation universitaire, le lieu de travail, les fonctions exercées au sein de l'entreprise, les intérêts, les contacts, et permettent le partage de données et de messages. En outre, de nombreux sites recensent les publications scientifiques indexées, notamment PubMed (National Library of Medicine, Bethesda [Maryland]), BioInfoBank Library (BioInfoBank Institute, Warsaw [Pologne]), CiteULike (Oversity Limited, Bristol [Angleterre]) et NextBio (NextBio, Santa Clara [Californie]).

En version bêta, Google vient de mettre en ligne Google Scholar Citations. Grâce à cet outil en ligne, vous pouvez sans frais et en quelques clics créer un profil professionnel de votre activité scientifique, à partir des publications recensées dans Google Scholar. Dès votre activation, l'outil propose l'ensemble des publications liées à votre nom; d'un coup d'œil rapide, vous pouvez retirer les publications non pertinentes et les doublons et publier de façon privée ou publique votre profil professionnel. L'outil permet un affichage convivial, dont une photo, les fonctions, les champs d'intérêt et la liste des publications qui peuvent être triées par titre, par premier auteur, par nombre de citations référencées et par année de publication. En outre, l'outil affiche le nombre de publications et le nombre de fois où les publications ont été citées depuis 2000 ainsi que le nombre de publications ayant été citées au moins dix fois depuis cinq ans. De plus, l'outil permet d'inviter tous les coauteurs de vos publications afin de faciliter le réseautage.

Comme BiomedExperts, Google Scholar Citations propose une liste préliminaire de vos publications recensées dans PubMed, mais aussi de toutes celles recensées par le moteur de Google Scholar. Ainsi, l'outil met en relief votre contribution à la vie professionnelle tant par vos publications indexées que par celles publiées dans des journaux professionnels (p. ex. le Journal canadien de la pharmacie hospitalière est publié dans PubMed Central, mais non indexé, tandis que le Pharmactuel est disponible en ligne en accès libre, mais non indexé).

Afin d'illustrer l'utilité des médias sociaux dans la vie professionnelle, nous décrivons brièvement l'expérience de notre unité de recherche en pratique pharmaceutique (URPP) au Centre hospitalier universitaire Sainte-Justine. Depuis janvier 2011, nous utilisons les médias sociaux afin de faciliter le transfert de connaissances et de joindre collègues et étudiants. L'URPP possède une page web à l'intérieur du site web de l'hôpital'. En complément à cette page statique, nous utilisons Wordpress afin de mettre hebdomadairement sur le blogue nos activités scientifiques ${ }^{10}$. La promotion de ce blogue se fait par Twitter, qui diffuse la nouvelle aux abonnés. Une page Facebook permet aux gens de manifester leur appui et leur intérêt pour les activités. Nous utilisons LinkedIn afin d'illustrer l'appartenance des membres à l'URPP, ce qui est utile notamment pour la mise à jour du CV des contributeurs. Enfin, Zotero (Roy Rosenzweig Center for History and New Media, Fairfax [Virginie]) nous permet de rendre accessibles et de regrouper nos publications.

$\mathrm{Au}$ cours de l'année 2011, plus de 50 commentaires présentant différents travaux scientifiques ont été ajoutés sur le blogue (Annexe 1). Le blogue permet non seulement de faire référence à des publications, mais aussi de publier les affiches présentées lors de congrès et de leur donner ainsi une seconde vie, qui fait suite à la séance d'affichage. Ces outils permettent enfin de garder le contact par le web et favorisent les collaborations interdisciplinaires.

Les pharmaciens et les médecins ont intérêt à utiliser les médias sociaux dans le cadre de leurs activités professionnelles. Google Scholar Citations offre un potentiel intéressant. L'expérience de l'URPP du CHU Sainte-Justine peut inspirer d'autres initiatives visant à favoriser la recherche évaluative et la diffusion des connaissances dans le domaine de la santé au moyen des médias sociaux.

\section{Références}

1. Pletneva N, Cruchet S, Simonet MA, Kajiwara M, Boyer C. Results of the $10 \mathrm{HON}$ survey on health and medical internet use. Stud Health Technol Inform 2011;169:73-77.

2. Greysen SR, Kind T, Chretien KC. Online professionalism and the mirror of social media. J Gen Intern Med 2010;25(11):1227-1229.

3. Kingsley K, Galbraith GM, Herring M, Stowers E, Stewart T, Kingsley KV. Why not just Google it? An assessment of information literacy skills in a biomedical science curriculum. BMC Med Educ 2011;11:17.

4. Williams J, Feild C, James K. The effects of a social media policy on pharmacy students' Facebook security settings. Am J Pharm Educ 2011; 75(9): 177 .

5. Kukreja P, Heck Sheehan A, Riggins J. Use of social media by pharmacy preceptors. Am J Pharm Educ 2011;75(9):176.

6. Farmer AD, Bruckner Holt CE, Cook MJ, Hearing SD. Social networking sites: a novel portal for communication. Postgrad Med J 2009;85(1007): 455-459.

7. Scanfeld D, Scanfled V, Larson EL. Dissemination of health information through social networks: Twitter and antibiotics. Am J Infect Control 2010;38(3):182-188.

8. Chou WY, Hunt YM, Beckjord EB, Moser RP, Hesse BW. Social media use in the United States: implications for health communication. Med Internet Res 2009; 11(4):e48.

9. L'Unité de recherche en pratique pharmaceutique (URPP). Montréal (QC) : CHU Sainte-Justine, Unité de recherche en pratique pharmaceutique; 2011. Publié au : www.chu-sainte-justine.org/urpp (consulté le 19 décembre 2011).

10. URPP : Qui sommes-nous? Montréal (QC) : CHU Sainte-Justine, Unité de recherche en pratique pharmaceutique; 2011. Publié au: http:// urppchusj.wordpress.com/ (consulté le 19 décembre 2011).

Jean-François Bussières, B. Pharm., M.SC., FCSHP

Chef

Département de pharmacie et Unité de recherche en pratique pharmaceutique

Denis Lebel, B. Pharm., M.Sc., FCSHP

Adjoint aux soins pharmaceutiques, à l'enseignement et à la recherche

Département de pharmacie et Unité de recherche en pratique pharmaceutique

Benoit Bailey, MD, FRCPC

Chef médical de l'urgence

CHU Sainte-Justine

Montréal, Québec

Jean-François Bussières est aussi professeur titulaire de clinique, Faculté de pharmacie, et Benoit Bailey est aussi toxicologue clinique, Département de pédiatrie, à I'Université de Montréal, Montréal, Québec. 
Annexe 1. Extrait web du blogue de I'Unité de recherche en pratique pharmaceutique (URPP) sur Wordpress.

URPP

Archiver dans la catégorie 'URPP'

\section{VendrediPM est maintenant disponible en anglais}

Comme promis, VendredipM est disponible en français et maintenant aussi en anqlais à l'enseigne http://fridaypm.ca. Ce site bilingue, en ligne depuis janvier 2011, est le premier site canadien à donner en temps réel la meilleure information disponible sur les ruptures d'approvisionnement de médicaments (pénurie de médicaments). Au cours des prochaines semaines, nous migrons à une mise en ligne en collaboration directe avec chacun des fabricants. Consultez l'onglet "ruptures en liqne".

\begin{tabular}{|c|c|}
\hline $\begin{array}{l}\text { Rédigé par cynthiatanguary } 1 \\
8 \text { dícembro } 2011 \text { a } 1503 \quad 23 \\
122312\end{array}$ & Publí dans URPP \\
\hline
\end{tabular}

\section{Utilisation de la glucarpidase dans les intoxications au méthotrexate}

La glucarpidase est un antidote qui peut être utile pour le traitement de l'intoxication au méthotrexate. Toutefois, l'optimisation de la pharmacothérapie préventive et la révision des feuilles d'ordonnances pré-rédigées s'imposent avant de considérer son utilisation. Pour les structures de soins amenées à traiter des patients par du méthotrexate à haute dose, il parait indispensable d'organiser l'approvisionnement du médicament de façon coordonnée entre les hôpitaux pour pouvoir obtenir rapidement la glucarpidase en cas d'intoxication au méthotrexate. Nul doute que le pharmacien doit assurer une veille électronique des résultats découlant des études en cours qu confirmeront ou pas, la place éventuelle de la glucarpidase en pratique clinique.

Vous pouvez consulter l'article publié dans le Bulletin d'Information Toxicoloqique de l'Institut national de santé publique du Québec.

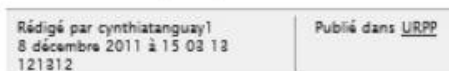

Avez-vous consulté la 18 e édition du Rapport canadien sur la pharmacie hospitalière?

Peu de professions de la santé ont le privilège d'avoir des données détaillées depuis plus de 3 décennies. Le comité éditorial sur la pharmacie hospitalière canadienne a publié en ligne au printemps 2011 le rapport 2009-2010; de plus, notre équipe de recherche a publié dans le Pharmactuel un article qui met en relief la pratique pharmaceutique hospitalière québécoise. Enfin, dans le cadre de la chronique d'une Page à l'autre de Québec Pharmacie, nous avons publié récemment un article court sur ces données. Alors que la pratique pharmaceutique est en pleine évolution, profitez de ces données probantes pour apprécier la contribution des pharmaciens, des assistants-techniques en pharmacie et du personnel de soutien en pharmacie hospitalière, à la prestation de services et soins pharmaceutiques, d'enseignement, de recherche et de gestion clinico-administrative. Bonne lecture! Vous pouvez consulter l'article paru dans le Pharmactuel.

Vous pouvez également consulter notre article publié dans le Québec Pharmacie. Cet article est publiế avec l'autorisation de l'éditeur de Québec Pharmacie.

\begin{tabular}{|c|c|}
\hline Rédigé par cynthiatanguay 1 , & Publió dans URPP \\
\hline
\end{tabular}

120112

\section{Étude comparative des organismes d'agrément en Europe, aux États-Unis et au Canada}

Une bonne compréhension des organismes d'agrément des établissements de santé à l'échelle internationale peut être utile à l'évolution optimale du circuit du médicament. L'objectif de cette étude descriptive était de comparer les organismes d'agrément au Canada, aux États-Unis et en Europe.

L'enquête du Belgian Hea/th Care Knowledge Centre (2008) présentait un état des lieux des organismes d'agrément en Europe et comportait 65 questions regroupées en cinq thématiques (i.e. policy. governance, methods, funding mechanisms, evaluation). La consultation des sites Internet de la foint Commission et d'Agrément Canada a permis de répondre à 26 des 65 questions de l'enquête européenne.

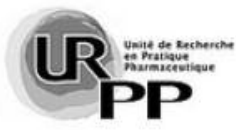

Pages

Pour an savoir plus

URPP sur le web

URPP sur CHUS!

URPP sur Facsbook

URPP sur Linkedin

URPP sur Twitter

URPP sur Zotorg

Archives

décembre 2011

novembre 2011

octobre 2011

septembre 2011

ivilat 2011

ivin 2011

mai 2011

avril 2011

mars 2011

Nouvelles de I'URPP

en format RSS

RS5 - Posts

Nos catégories

$\underline{\text { URPP (44) }}$ 